\title{
Convergence of the Eckmann and Ruelle Algorithm for the Estimation of Liapunov Exponents.
}

\author{
M. Eugenia Mera. Manuel Morán. \\ Departamento de Análisis Económico. Universidad Complutense. \\ Campus de Somosaguas. 28223 Madrid. España. \\ E-Mail Address: ececo06@sis.ucm.es
}

\begin{abstract}
We analyze the convergence conditions of the Eckmann and Ruelle algorithm (E.R.A. for the sequel) used to estimate the Liapunov exponents, for the tangent map, of an ergodic measure, invariant under a smooth dynamical system. We find sufficient conditions for this convergence which are related to those ensuring the convergence to the tangent map of the best linear $L^{p}$-fittings of the action of a mapping $f$ on small balls. Under such conditions, we show how to use E.R.A. to obtain estimates of the Liapunov exponents, up to an arbitrary degree of accuracy. We propose an adaptation of E.R.A. for the computation of Liapunov exponents in smooth manifolds which allows us to avoid the problem of detecting the spurious exponents.

We prove, for a Borel measurable dynamics $f$, the existence of Liapunov exponents for the function $S_{r}(x)$, mapping each point $x$ to the matrix of the best linear $L^{p}$-fitting of the action of $f$ on the closed ball of radius $r$ centered at $x$, and we show how to use E.R.A. to get reliable estimates of the Liapunov exponents of $S_{r}$. We also propose a test for checking the differentiability of an empirically observed dynamics.

Keywords and Phrases: Liapunov exponents, Eckmann and Ruelle algorithm, best $L^{p}$-linear estimate, nonlinear dynamics, invariant and ergodic measures.

Partially supported by Dirección Central de Investigación Científica y Técnica. PS95-0193.
\end{abstract}




\section{Introduction.}

In this article we find conditions ensuring the convergence of the Eckmann and Ruelle algorithm (see [7]) used in the estimation of the Liapunov exponents, for the tangent map, of an ergodic measure, invariant under a smooth dynamical system. We start by introducing the Liapunov exponents.

\subsection{Liapunov exponents of dynamical systems. Oseledec theorem.}

Let $f: M \rightarrow M$ be a dynamical system where $M$ is a Borel subset of $\mathrm{IR}^{s}$, and let $\mu$ be an $f$-invariant probability measure on the $\sigma$-algebra of Borel subsets of $M$. Let $S: M \rightarrow \mathcal{M}_{s}$ be a measurable map where $\mathcal{M}_{s}$ is the set of real $s \times s$ matrices and $S$ is such that $\log ^{+}(\|S(x)\|) \in L^{1}(\mu)$ (for an arbitrary matrix $A$, we denote by $\|A\|$ the largest eigenvalue of $\left(A^{*} A\right)^{1 / 2}$ where $A^{*}$ denotes the transposed matrix of $A$ ). Consider the matrix $S_{z}^{(q)}$ defined by

$$
S_{z}^{(q)}=S\left(f^{q-1}(z)\right) S\left(f^{q-2}(z)\right) \cdots S(z),
$$

where $f^{k}$ denotes the $k$-fold composition $f \circ f \circ \cdots \circ f$. The multiplicative ergodic theorem proved by Oseledec (see [13]) states that the limit

$$
\Lambda_{z}=\lim _{q \rightarrow \infty}\left(S_{z}^{(q) *} S_{z}^{(q)}\right)^{(2 q)^{-1}}
$$

exists $\mu$-a.e. The logarithms of the eigenvalues of $\Lambda_{z}$ are called characteristic (or Liapunov) exponents of $S$ at $z$. If $\mu$ is ergodic, they are constant $\mu$-a.e.

If $M$ is a smooth submanifold of $\operatorname{IR}^{s}, f \in C^{1}(M)$ and $\mu$ has a compact support, the Liapunov exponents for $S(x)=D f(x)$ are well defined (see [15]). They quantify the sensitivity of the system to initial conditions, and give relevant information about the entropy and fractal dimension of the invariant measure $\mu$ (see [6]). The eigenspaces of the matrix $\Lambda_{z}$ also give useful information on the local structure of $\mu$.

\subsection{Estimation of the Liapunov exponents of an observed dynamics. The Eckmann and Ruelle algorithm.}

Assume that the state space $M$ defined in section 1.1. is an open subset of $\mathrm{IR}^{s}$. When computing the Liapunov exponents of $D f$ a standard problem arises when 
the time one mapping $f$ is unknown and the tangent map must be estimated by observing a given vectorial time series $\left\{x_{0}, \ldots, x_{n-1}\right\} \subset \operatorname{IR}^{s}$. This time series is assumed to consist of the first $n$ points of the orbit of the initial state $z$ under an unknown evolution law $f$ of a dynamical system, i.e. $x_{i}=f^{i}(z), i=0, \ldots, n-1$ (we denote this time series by $O_{n}(z)$ ). Most of the algorithms used to estimate Liapunov exponents use a linear fitting of the tangent map (see for instance [7], [16] and [1]). One of the most frequently used is E.R.A. It has proved computationally efficient in giving the whole Liapunov spectrum of many dynamics, instead of the largest Liapunov exponent, as done by other standard algorithms ([21], [17]). However we do not know of any rigorous proof of its convergence. We provide it in Theorem 2.3. In particular we show how E.R.A. may be used to compute the whole Liapunov spectrum of a smooth dynamics, up to an arbitrary degree of accuracy. We now describe how this algorithm works.

Let $D f_{z}^{(q)}$ be the matrix defined by (1.1) for $S=D f$. Since $D f$ is unknown, the algorithm replaces it by an estimate. Given $x_{i} \in O_{n}(z)$, the estimate of $D f\left(x_{i}\right)$ is

obtained by taking the linear map $\beta_{r, x_{i}, n}$ which best describes how the evolution law takes the vectors $x_{j}-x_{i}$ to the vectors $x_{j+1}-x_{i+1}$ for vectors $x_{j}$ in a small ball centered at $x_{i}$. Given a radius $r>0$, let $N_{r, n}(i)$ denote the set of indices $j$ such that $\left|x_{j}-x_{i}\right|_{2} \leq r\left(|\cdot|_{2}\right.$ denotes the Euclidean norm) and $x_{j} \in O_{n}(z)$. If we use the least squares fit then $\beta_{r, x_{i}, n}$ minimizes, in the set of linear maps

$$
\mathcal{A}_{r, x_{i}, n}(\beta):=\left[\frac{1}{n} \sum_{j \in N_{r, n}(i)}\left(\left|x_{j+1}-x_{i+1}-\beta\left(x_{j}-x_{i}\right)\right|_{2}\right)^{2}\right]^{1 / 2}
$$

In order to obtain the convergence of the algorithm we need to ensure the goodness of the estimate $\beta_{r, x_{i}, n}$ of $D f\left(x_{i}\right)$.

\subsection{Convergence of E.R.A: description of the proof.}

We assume from now onwards that we work in the setting described in section 1.1. Given a point $x$ in $M$, let $B(x, r)$ denote the closed ball, in the Euclidean norm, with radius $r$ centered at $x$. Given $p$ and $r$, with $1<p<\infty$ and $r>0$ we define, on the set $\mathcal{L}_{s}$ of linear maps from $\mathrm{IR}^{s}$ to $\mathrm{IR}^{s}$, the functional

$$
\mathcal{A}_{r, x, \mu}(\beta):=\left[\int_{B(x, r)}\left(|f(y)-f(x)-\beta(y-x)|_{p}\right)^{p} d \mu(y)\right]^{1 / p},
$$


where $|\cdot|_{p}$ is the usual $p$-norm of vectors in $\operatorname{IR}^{s}$. If there is a unique $\beta_{r, x, \mu} \in \mathcal{L}_{s}$ where the minimum of $\mathcal{A}_{r, x, \mu}$ is attained, we say that $\beta_{r, x, \mu}$ is the best linear estimate in $L^{p}(\mu \mid B(x, r))$-norm of $f$ at $x$ (throughout the text $\mu \mid B(x, r)$ denotes the restriction of the measure $\mu$ to the ball $B(x, r))$.

Notice that (1.3) coincides with (1.2) when $p=2, x=x_{i}$ and $\mu=\mu_{z, n}$

with $\mu_{z, n}=n^{-1} \sum_{j=0}^{n-1} \delta_{f^{j}(z)}$. Thus, E.R.A. replaces $D f\left(x_{i}\right)$ with the linear map $\beta_{r, x_{i}, \mu_{z, n}}$ which is the best linear estimate in $L^{p}\left(\mu_{z, n} \mid B\left(x_{i}, r\right)\right)$-norm of $f$ at $x_{i}$. If $f$ is a Borel measurable dynamics, and $\mu$ is an $f$-invariant and ergodic measure, we prove that $\lim _{n \rightarrow \infty} \beta_{r, x_{i}, \mu_{z, n}}=\beta_{r, x_{i}, \mu} \mu$-a.e. $z \in M$, where $\beta_{r, x_{i}, \mu}$ is the best linear estimate in $L^{p}\left(\mu \mid B\left(x_{i}, r\right)\right)$-norm of $f$ at $x_{i}$. The goodness of $\beta_{r, x_{i}, \mu_{z, n}}$ as an estimate of $D f\left(x_{i}\right)$ may thus be obtained by proving first the goodness of $\beta_{r, x_{i}, \mu_{z, n}}$ as an estimate of $\beta_{r, x_{i}, \mu}$, and then the goodness of $\beta_{r, x_{i}, \mu}$ as an estimate of $\operatorname{Df}\left(x_{i}\right)$.

The determination of sufficient conditions for the convergence of $\beta_{r, x, \mu}$ to $D f(x)$ when the radius $r$ tends to zero is not a trivial problem due to the fact that the measure $\mu$ may exhibit a complex local structure. It is shown in [11] that there exists an inverse relationship between the regularity conditions of $\mu$, in terms of local densities, and the smoothness of the mapping $f$ required to ensure the goodness of the estimates. Also, if the measure $\mu \mid B(x, r)$, for small $r$, is concentrated near a hyperplane, $\beta_{r, x, \mu}$ may be a poor estimate. This is known to happen when estimating the Liapunov exponents of an empirically recorded time series with embedding techniques (see [18], [7]). In that case, the inaccuracy of linear fittings makes it difficult to distinguish between the exponents given by the algorithm which are the true Liapunov exponents of the measure and those which are spurious. We discuss how to get round this problem in section 1.6.

\section{4. $\mathbf{L}_{r}^{p}$-Liapunov exponents.}

Assume now that the point to matrix function $S_{r, \mu}$ mapping each point $x$ to the matrix of the best linear estimate in $L^{p}(\mu \mid B(x, r))$-norm of $f$ at $x$ is well defined. We may then ask if the hypotheses of the Oseledec theorem hold and hence the Liapunov exponents of $S_{r, \mu}$ are well defined. If they are, we call them $\mathbf{L}_{r}^{p}$-Liapunov exponents. The interpretation of the Liapunov exponents of $D f$ as asymptotic exponential rates of convergence or divergence of infinitely nearby orbits is obtained by applying the chain rule to $D f^{q} v$, where $v$ is a perturbation in the tangent space. However, in practice we may not have access to infinitely nearby orbits and the map $S_{r, \mu}(x)$ describes the evolution of observable data, in the ball $B(x, r)$, better than $D f(x)$ does. Although the chain rule does not work 
for the linear $L_{r}^{p}$-estimates, the Oseledec theorem provides a multiplicative average of the $S_{r, \mu}(x)$ matrices along the orbit. The interest of the $L_{r}^{p}$-Liapunov exponents lies in the fact that they are also defined for Borel measurable non-differentiable dynamics, and the state space $M$ may be any Borel subset of $\operatorname{IR}^{s}$ (see Theorem 2.1).

To compute the $L_{r}^{p}$-Liapunov exponents we have to modify E.R.A. slightly, keeping the radii of the balls fixed, instead of taking a given number of closest neighbours as in the original algorithm. In particular, if we keep the radius $r$ of the balls constant, take a sufficiently large number $q$ of matrices to be estimated, and a sufficiently large number $n>>q$ of points in the orbit, this version of E.R.A. gives us the $L_{r}^{p}$-Liapunov exponents up to an arbitrary degree of accuracy (see Theorem 2.2). If instead we first take a sufficiently large $q$, then fix a sufficiently small value of $r$ and then take an $n$ large enough, we can obtain the Liapunov spectrum of $D f$, for a smooth dynamics $f$, with arbitrary accuracy.

In order to reduce the amount of time needed to make linear fits using all points in each ball we can incorporate the box-assisted device for finding neighboring points (see [8]). See [12] for a more detailed discussion of the practical issues involved in the use of the algorithm.

\subsection{Test for the smoothness of the dynamics.}

We need to check the smoothness of empirical data when computing their Liapunov exponents, if we want to interpret them as estimates of the Liapunov spectrum of the tangent map. To this end we provide an additive test to measure the degree of differentiability of the data. Let $\mathcal{A}_{r, x, \mu}$ be defined by (1.3) and let $S_{r, \mu}$ be the function mapping each point $x$ to the matrix $S_{r, \mu}(x)$ of the best linear estimate in $L^{p}(\mu \mid B(x, r))$-norm of $f$ at $x$. The weighted error corresponding to $S_{r, \mu}(x)$ is $(\mu(B(x, r)))^{-1 / p} \mathcal{A}_{r, x, \mu}\left(S_{r, \mu}(x)\right)$. The goodness of the linear fits is defined by

$$
\begin{gathered}
R_{r, \mu}\left(S_{r, \mu}\right)=\int_{M} \frac{1}{(\mu(B(x, r)))^{1 / p}} \mathcal{A}_{r, x, \mu}\left(S_{r, \mu}(x)\right) d \mu(x)= \\
\int_{M}\left(\frac{1}{\mu(B(x, r))} \int_{B(x, r)}\left(\left|f(y)-f(x)-S_{r, \mu}(x)(y-x)\right|_{p}\right)^{p} d \mu(y)\right)^{1 / p} d \mu(x) .
\end{gathered}
$$

In Theorem 2.1. we prove that under suitable conditions this quantity is well defined. Since the algorithm replaces $S_{r, \mu}(x)$ with $S_{r, \mu_{z, n}}(x)$ where $\mu_{z, n}=$ $n^{-1} \sum_{j=0}^{n-1} \delta_{f^{j}(z)}$ (see section 1.3), the algorithm gives 


$$
R_{r}^{(q)}\left(S_{r, \mu_{z, n}}\right):=\frac{1}{q} \sum_{i=0}^{q-1} \frac{1}{\left(\mu_{z, n}\left(B\left(f^{i}(z), r\right)\right)\right)^{1 / p}} \mathcal{A}_{r, f^{i}(z), \mu_{z, n}}\left(S_{r, \mu_{z, n}}\left(f^{i}(z)\right)\right)
$$

as estimate of $R_{r, \mu}\left(S_{r, \mu}\right)$. In Theorem $\mathbf{2 . 2}$ we prove the convergence of $R_{r}^{(q)}\left(S_{r, \mu_{z, n}}\right)$ to $R_{r, \mu}\left(S_{r, \mu}\right)$ when $q$ and $n$ tend to infinity. Finally, we define the parameter

$$
R:=\liminf \operatorname{in}_{r \rightarrow 0} \frac{\log \left(R_{r, \mu}\left(S_{r, \mu}\right)\right)}{\log r}
$$

and in proposition 1 we provide lower bounds for $R$ when $f$ is a Hölder, a Lipschitz, or a $C^{1+\varepsilon}$ function. In particular, in the differentiable case we prove that $R \geq 1$.

\subsection{Adaptation of E.R.A. to a dynamics in a smooth submanifold of IR $^{s}$.}

In Theorem 2.3 we give sufficient conditions for the convergence of E.R.A. to the Liapunov exponents of the tangent map $D f$ when a smooth dynamics $f$ is defined on a $d$-dimensional smooth submanifold $M$ in $\mathrm{IR}^{s}$, with $d \leq s$. We briefly describe how the algorithm works in this case. Let $\left(U_{i}, \Phi_{i}\right)$ be a chart at $x_{i} \in$ $O_{n}(z)$ with $\Phi_{i}\left(x_{i}\right)=0$, and let $g_{i}=\Phi_{i+1} \circ f \circ \Phi_{i}^{-1}$. Then, $D f\left(x_{i}\right)$ is defined as $D f\left(x_{i}\right)=D \Phi_{i+1}^{-1}(0) D g_{i}(0) D \Phi_{i}\left(x_{i}\right)$ and is independent of the chosen charts. The Liapunov exponents are the logarithms of the eigenvalues of the matrix $\Lambda_{z}=$ $\lim _{q \rightarrow \infty}\left(D g^{(q) *} D g^{(q)}\right)^{(2 q)^{-1}}$ where $D g^{(q)}=D g_{q-1}(0) D g_{q-2}(0) \cdots D g_{0}(0)$ and do not depend on the chosen charts either. The problem is how to select charts $\left(U_{i}, \Phi_{i}\right)$ at the points $x_{i} \in O_{n}(z)$ and, given the choice, to prove the convergence to $D g_{i}(0)$ of the best linear estimate in $L^{p}\left(\nu_{n, i} \mid B(0, r)\right)$-norm of $g_{i}$ at the origin, where $\nu_{n, i}:=\Phi_{i} \#\left(\mu_{z, n} \mid U_{i}\right)$ is the measure induced by $\mu_{z, n} \mid U_{i}$ under the map $\Phi_{i}$, and $\mu_{z, n}=n^{-1} \sum_{j=0}^{n-1} \delta_{f^{j}(z)}$. Let $x_{i_{j}} \in O_{n}(z), j=1, \ldots, d$ be the nearest neighbours of the point $x_{i}$ such that the vectors $v_{j}=x_{i_{j}}-x_{i}, j=1, \ldots, d$ are linearly independent, and let $T_{i}$ be the subspace spanned by them. The algorithm takes an $r_{0}>0$, and a chart $\left(U_{i}, \Phi_{i}\right)$ at $x_{i}$, where $U_{i}=\left\{y \in \mathrm{IR}^{s}:\left|y-x_{i}\right|_{2}<r_{0}\right\}$, such that the restriction of $\Phi_{i}$ to $U_{i} \cap O_{n}(z)$ is given by $\Phi_{i}(x)=\Pi_{T_{i}}\left(x-x_{i}\right)$, where $\Pi_{T_{i}}$ denotes the orthogonal projection of $\operatorname{IR}^{s}$ on $T_{i}$. We show in Theorem 2.3 that such a chart exists for small enough $r_{0}$. Notice that in this case the estimate of $D g^{(q)}$ is a $d \times d$ matrix. Therefore this method gives an estimate of the $d$ true exponents of $D f$ thus avoiding the often pointed out problem (see [2], [7] and [16]) of detecting the $s-d$ spurious exponents. 


\section{Results.}

We work in the framework described in section 1.1. Recall that $\mathcal{L}_{s}$ denotes the set of linear maps from $\mathrm{IR}^{s}$ to $\mathrm{IR}^{s}, \mathcal{M}_{s}$ denotes the set of real $s \times s$ matrices, and $Q^{*}$ stands for the transposed matrix of the matrix $Q$. We denote by $\operatorname{spt}(\mu)$ the topological support of $\mu$ and by $\operatorname{dim} \mu$ the Hausdorff dimension of the measure $\mu$ defined by $\operatorname{dim} \mu=\inf \{\operatorname{dim}(A): \mu(A)>0\}$ where $\operatorname{dim}(A)$ is the Hausdorff dimension of the set $A$ (see [10]).

We start with a lemma which states sufficient conditions on $f, \mu$ and $p$ for the existence and uniqueness of the best linear estimate in $L^{p}(\mu \mid B(x, r))$-norm of $f$ at the point $x \in M$ where $M$ is a Borel subset of $\operatorname{IR}^{s}$. In order to obtain uniqueness we need to assume that $\mu \mid B(x, r)$ is not concentrated on hyperplanes. We denote by $P(B(x, r))$ the set of Borel probability measures on $M$ such that $\mu(H \cap B(x, r))<$ $\mu(B(x, r))$ holds for any hyperplane $H$. We adopt the notation $\|\beta\|_{2}$ for the usual norm of linear maps, that is $\|\beta\|_{2}=\max \left\{|\beta v|_{2}:|v|_{2}=1, v \in \operatorname{IR}^{s}\right\}$.

For $x \in M, r>0, \mu \in P(B(x, r))$ and $p \in(1, \infty)$ we define, on the set $\mathcal{L}_{s}$, the functional $\mathcal{A}_{r, x, \mu}$ given by (1.3) and the functional $h_{r, x, \mu}$ given by

$$
h_{r, x, \mu}(\beta)=\left[\int_{B(x, r)}\left(|\beta(y-x)|_{p}\right)^{p} d \mu(y)\right]^{1 / p} .
$$

Lemma 1. Let $\mathcal{S}=\left\{\beta \in \mathcal{L}_{s}:\|\beta\|_{2}=1\right\}, p \in(1, \infty), x \in M, r>0$, and $\mu \in P(B(x, r))$. Then

(i) There is a $T_{r, x, \mu} \in \mathcal{S}$ where the minimum value of $h_{r, x, \mu}$ on $\mathcal{S}$ is attained and $h_{r, x, \mu}\left(T_{r, x, \mu}\right)>0$.

(ii) Let $|f|_{p} \in L^{p}(\mu \mid B(x, r))$. Then, there is a unique $\beta_{r, x, \mu} \in \mathcal{L}_{s}$ where the minimum value of $\mathcal{A}_{r, x, \mu}$ on $\mathcal{L}_{s}$ is attained and

$$
\left\|\beta_{r, x, \mu}\right\|_{2} \leq \frac{2\left[\int_{B(x, r)}\left(|f(y)-f(x)|_{p}\right)^{p} d \mu(y)\right]^{1 / p}}{h_{r, x, \mu}\left(T_{r, x, \mu}\right)} .
$$

Proof. The first statement of part (i) follows from the continuity of the functional $h_{x, r, \mu}$ on the compact set $\mathcal{S}$, and the assumption $\mu \in P(B(x, r))$ ensures $h_{r, x, \mu}\left(T_{r, x, \mu}\right)>0$.

Since $|f|_{p} \in L^{p}(\mu \mid B(x, r)), \mathcal{A}_{r, x, \mu}(\alpha)<\infty$ for every $\alpha \in \mathcal{L}_{s}$. Let $\tau:=\inf _{\alpha \in \mathcal{L}_{s}} \mathcal{A}_{r, x, \mu}(\alpha)$, and $R:=\frac{\tau+\mathcal{A}_{r, x, \mu}(0)}{h_{r, x, \mu}\left(T_{r, x, \mu}\right)}$. Then $\mathcal{A}_{r, x, \mu}(\alpha)>\tau$ if $\|\alpha\|_{2}>R$, so that the continuous functional $\mathcal{A}_{r, x, \mu}$ attains its minimum in $\mathcal{L}_{s}$. The uniqueness of the minimum 
can be obtained from the strict convexity of the normed space $L^{p}(\mu \mid B(x, r))$ for $p \in(1, \infty)$ and from the fact $\mu \in P(B(x, r))$. Let $T_{r, x, \mu} \in \mathcal{S}$ be the linear map obtained in (i). Then, for any $\alpha \in \mathcal{L}_{s}, h_{r, x, \mu}(\alpha) \geq\|\alpha\|_{2} h_{r, x, \mu}\left(T_{r, x, \mu}\right)$ holds, which for $\alpha=\beta_{r, x, \mu}$ gives

$$
\left\|\beta_{r, x, \mu}\right\|_{2} \leq \frac{h_{r, x, \mu}\left(\beta_{r, x, \mu}\right)}{h_{r, x, \mu}\left(T_{r, x, \mu}\right)} \leq \frac{\mathcal{A}_{r, x, \mu}\left(\beta_{r, x, \mu}\right)+\left[\int_{B(x, r)}\left(|f(y)-f(x)|_{p}\right)^{p} d \mu(y)\right]^{1 / p}}{h_{r, x, \mu}\left(T_{r, x, \mu}\right)}
$$

This inequality together with $\mathcal{A}_{r, x, \mu}\left(\beta_{r, x, \mu}\right) \leq \mathcal{A}_{r, x, \mu}(0)$ completes the proof of $(2.2)$.

The following theorem establishes the existence of $L_{r}^{p}$-Liapunov exponents. In a previous version, this theorem was proved for a continuous dynamics. The present formulation for a measurable dynamics, in the spirit of the Oseledec theorem, has been possible due to the observation of an anonymous referee that $\mathcal{A}_{r, x, \mu}(S)$ is a smooth functional on $S$. This has also substantially simplified the proof of the theorem.

Theorem 2.1. Let $M$ be a Borel subset of $\operatorname{IR}^{s}$ and let $f: M \rightarrow M$ be a Borel measurable dynamics. Let $\mu$ be an $f$-invariant measure with compact support and $\operatorname{dim} \mu>s-1$. For $p \in(1, \infty)$ and $r>0$, let $S_{r, \mu}: \operatorname{spt}(\mu) \rightarrow \mathcal{M}_{s}$ be the function which maps each point $x \in \operatorname{spt}(\mu)$ to the matrix of the best linear estimate in $L^{p}(\mu \mid B(x, r))$-norm of $f$ at $x$, i.e. $S_{r, \mu}(x)=\beta_{r, x, \mu}$. Then

(i) The limit $\Gamma_{r, \mu}(z):=\lim _{q \rightarrow \infty}\left(S_{r, \mu, z}^{(q) *} S_{r, \mu, z}^{(q)}\right)^{(2 q)^{-1}}$ exists $\mu$-a.e., where $S_{r, \mu, z}^{(q)}$ is defined as in (1.1) for $S=S_{r, \mu}$.

(ii) Let $\alpha_{r, \mu, 1}(z)>\alpha_{r, \mu, 2}(z)>\cdots>\alpha_{r, \mu, k}(z)$ be the logarithms of the non equal eigenvalues of $\Gamma_{r, \mu}(z)$ (we call them $\boldsymbol{L}_{r}^{p}$-Liapunov exponents at the point $z$ ) and $E_{j}(z)$ be the subspace generated by the eigenvectors corresponding to those eigenvalues of $\Gamma_{r, \mu}(z)$ not larger than $\exp \left(\alpha_{r, \mu, j}(z)\right)$. Then $E_{1}(z) \supset \cdots \supset E_{k}(z)$ and for $v \in E_{j}(z) \backslash E_{j+1}(z)$

$$
\lim _{q \rightarrow \infty} q^{-1} \log \left|S_{r, \mu, z}^{(q)}(v)\right|_{2}=\alpha_{r, \mu, j}(z) .
$$

(iii)The functions $z \rightarrow \alpha_{r, \mu, j}(z)$ and $z \rightarrow \operatorname{dim}\left(E_{j}(z)\right)$ are measurable and $f$ invariant. If $\mu$ is ergodic, they are constant $\mu$-a.e.

(iv) The quantity $R_{r, \mu}\left(S_{r, \mu}\right):=\int_{M} \frac{1}{\left(\mu(B(x, r))^{1 / p}\right.} \mathcal{A}_{r, x, \mu}\left(S_{r, \mu}(x)\right) d \mu(x)$ is well defined. If $\mu$ is ergodic, then $R_{r, \mu}\left(S_{r, \mu}\right)=\lim _{q \rightarrow \infty} R_{r}^{(q)}\left(S_{r, \mu}\right)$ holds for $\mu$-a.e. $z \in M$, where $R_{r}^{(q)}\left(S_{r, \mu}\right)=\frac{1}{q} \sum_{i=0}^{q-1} \frac{1}{\left(\mu\left(B\left(f^{i}(z), r\right)\right)\right)^{1 / p}} \mathcal{A}_{r, f^{i}(z), \mu}\left(S_{r, \mu}\left(f^{i}(z)\right)\right)$. 
Proof. To prove statements (i) to (iii) we show that the hypotheses of Oseledec theorem hold for the dynamics restricted to the full $\mu$-measure set $M^{*}=$ $\cap_{i=0}^{\infty} f^{-i}(\operatorname{spt}(\mu))$ and for the matrix valued mapping $S_{r, \mu}$. Since $\operatorname{dim} \mu>s-1$, we have that $\mu \in P(B(x, r))$ for any $x \in \operatorname{spt}(\mu)$. Using the compactness of $\operatorname{spt}(\mu)$ we get $|f|_{p} \in L^{\infty}(\mu)$, so in particular that $|f|_{p} \in L^{p}(\mu)$, and by Lemma 1 the function $S_{r, \mu}$ is defined on $\operatorname{spt}(\mu)$ and $S_{r, \mu}\left(f^{i}(z)\right)$ is defined for every $z \in M^{*}, i \in \mathrm{IN}$.

Let $\left\{x_{n}\right\}$ be a sequence on $\operatorname{spt}(\mu)$ converging to $x$. Since $\mu(\partial B(x, r))=0$ for any $x \in M$ and $r>0$, we get the weak convergence of the sequence of measures $\left\{\mu \mid B\left(x_{n}, r\right)\right\}$ to $\mu \mid B(x, r)$. From this, the continuity of the function $H$ defined on the compact set $\operatorname{spt}(\mu) \times \mathcal{S}$ by $H(x, T)=h_{r, x, \mu}(T)$ can be derived (recall that $\left.\mathcal{S}=\left\{T \in \mathcal{L}_{s}:\|T\|_{2}=1\right\}\right)$. Then, there is a $\left(x^{*}, T^{*}\right) \in \operatorname{spt}(\mu) \times \mathcal{S}$ where $H$ attains its minimum value, and using (i) of Lemma 1 we have that $H\left(x^{*}, T^{*}\right)>0$. Using this fact together with (2.2) we can get a positive constant $K$ such that $\left\|S_{r, \mu}(x)\right\|_{2}<K$ for $x \in \operatorname{spt}(\mu)$.

Since the map $S \rightarrow \mathcal{A}_{r, x, \mu}(S)$ is a convex and differentiable functional in the set $G=\left\{\beta \in \mathcal{L}_{s}:\|\beta\|_{2}<K\right\}$, and the unique minimum $S_{r, \mu}(x)$ of $\mathcal{A}_{r, x, \mu}$ belongs to $G$, the map $S_{r, \mu}$ is unambiguously characterized (see [14]) by $\psi(x, S)=0$ where $\psi$ is the Borel measurable function defined on $M^{*} \times \operatorname{IR}^{s^{2}}$ by $\psi(x, S)=$ $\left|\operatorname{Grad}_{r, x, \mu}(S)\right|_{2}$. Thus, the graph of $S_{r, \mu}$

$$
\operatorname{Graph}\left(S_{r, \mu}\right):=\left\{\left(x, S_{r, \mu}(x)\right): x \in M^{*}\right\}=\left\{(x, S) \in M^{*} \times \operatorname{IR}^{s^{2}}: \psi(x, S)=0\right\}
$$

belongs to the $\sigma$-field of the Borel subsets of $M^{*} \times \mathrm{IR}^{s^{2}}$ and then (see Theorem 2.3 and Remark in pp. 7 of [5]) $S_{r, \mu}$ is Borel measurable on $M^{*}$. The proof of statements (i) to (iii) is completed by proving that $\log ^{+}\left(\left\|S_{r, \mu}(x)\right\|_{2}\right) \in L^{1}(\mu)$ which is obtained using that $\left\|S_{r, \mu}(x)\right\|_{2}$ is bounded in $\operatorname{spt}(\mu)$.

The proof of (iv) follows from Birkhoff's Ergodic theorem (see [20]). It suffices to check that the map $G(x)=\frac{1}{(\mu(B(x, r)))^{1 / p}} \mathcal{A}_{r, x, \mu}\left(S_{r, \mu}(x)\right)$ is $\mu$-integrable. The Borel measurability is obtained by the measurability of the functions $x \rightarrow \mu(B(x, r))$, $(x, S) \rightarrow \mathcal{A}_{r, x, \mu}(S)$ and $x \rightarrow S_{r, \mu}(x)$. The integrability can then be proved by taking into account that the maps $x \rightarrow\left\|S_{r, \mu}(x)\right\|_{2}$ and $x \rightarrow|f(x)|_{p}$ belong to $L^{\infty}(\mu)$.

The next theorem shows how E.R.A. can be used to compute the $L_{r}^{p}$-Liapunov exponents. Given a finite orbit $O_{n}(z)=\left\{f^{i}(z): i=0, \ldots, n-1\right\}$, the natural approximation for $\mu$ from $O_{n}(z)$ is $\mu_{z, n}=n^{-1} \sum_{i=0}^{n-1} \delta_{f^{i}(z)}$. Thus, the algorithm acts by fitting the matrix $S_{r, \mu_{z, n}}(x)$ of the best linear estimate in $L^{p}\left(\mu_{z, n} \mid B(x, r)\right)$-norm 
of $f$ at $x \in O_{n}(z)$. Assume that $S_{r, \mu_{z, n}}(x)$ is defined for any $x \in O_{n}(z)$, let $S_{r, \mu_{z, n}, z}^{(q)}$ be defined as in (1.1) for $S=S_{r, \mu_{z, n}}$ and let $\Gamma_{r, \mu_{z, n}, q}(z)=\left(S_{r, \mu_{z, n}, z}^{(q) *} S_{r, \mu_{z, n},}^{(q)}\right)^{\frac{1}{2 q}}$. Then, the exponents $\alpha_{r, \mu_{z, n}, j}^{(q)}, j=1, \ldots, s$ that E.R.A. gives, are the logarithms of the eigenvalues of the matrix $\Gamma_{r, \mu_{z, n}, q}(z)$. The estimate of $R_{r, \mu}\left(S_{r, \mu}\right)$ given by the algorithm is

$$
R_{r}^{(q)}\left(S_{r, \mu_{z, n}}\right):=\frac{1}{q} \sum_{i=0}^{q-1} \frac{1}{\left(\mu_{z, n}\left(B\left(f^{i}(z), r\right)\right)\right)^{1 / p}} \mathcal{A}_{r, f^{i}(z), \mu_{z, n}}\left(S_{r, \mu_{z, n}}\left(f^{i}(z)\right)\right) .
$$

Theorem 2.2. Let $M$ be a Borel subset of $\mathrm{IR}^{s}$, and let $f: M \rightarrow M$ be a Borel measurable dynamics. Let $\mu$ be an $f$-invariant and ergodic measure with compact support and $\operatorname{dim} \mu>s-1$. For $p \in(1, \infty)$ and $r>0$, let $\alpha_{r, \mu, j}, j=1, \ldots, s$ be the $\mu$-a.e. constant $L_{r}^{p}$-Liapunov exponents. Given $O_{n}(z)=\left\{f^{i}(z): i=0, \ldots, n-1\right\}$, let $\alpha_{r, \mu_{z, n}, j}^{(q)}(z), j=1, \ldots, s$ be the estimates of the $L_{r}^{p}$-Liapunov exponents obtained by the Eckmann and Ruelle algorithm from $O_{n}(z)$. Then,

$$
\lim _{q \rightarrow \infty} \lim _{n \rightarrow \infty} \alpha_{r, \mu_{z, n}, j}^{(q)}(z)=\alpha_{r, \mu, j}, j=1, \ldots, s
$$

and

$$
\lim _{q \rightarrow \infty} \lim _{n \rightarrow \infty} R_{r}^{(q)}\left(S_{r, \mu_{z, n}}\right)=R_{r, \mu}\left(S_{r, \mu}\right)
$$

for $\mu$-a.e. $z \in M$.

Proof. By Theorem 2.1, for any $p \in(1, \infty)$ and $r>0$ the $L_{r}^{p}$-Liapunov exponents $\alpha_{r, \mu, j}, 1 \leq j \leq s$ are defined, and by the ergodicity of $\mu$ they are constant $\mu$ a.e. They are the logarithms of the eigenvalues of the $\mu$-a.e well defined matrix $\Gamma_{r, \mu}(z):=\lim _{q \rightarrow \infty} \Gamma_{r, \mu, q}(z)$ where $\Gamma_{r, \mu, q}(z):=\left(S_{r, \mu, z}^{(q) *} S_{r, \mu, z}^{(q)}\right)^{(2 q)^{-1}}$ and $S_{r, \mu, z}^{(q)}$ is defined as in (1.1) for $S=S_{r, \mu}$. Since the characteristic polynomial of any matrix is a continuous function of its entries and the roots of any polynomial are continuous functions of its coefficients (see [9]), it follows that the eigenvalues of any matrix are continuous functions of its entries and then

$$
\lim _{q \rightarrow \infty} \alpha_{r, \mu, j}^{(q)}(z)=\alpha_{r, \mu, j}, j=1, \ldots, s
$$

where $\alpha_{r, \mu, j}^{(q)}(z)$ are the logarithms of the eigenvalues of $\Gamma_{r, \mu, q}(z)$.

The exponents computed by E.R.A., that we have denoted by $\alpha_{r, \mu_{z, n}, j}^{(q)}(z)$, $j=1, \ldots, s$, are the logarithms of the eigenvalues of the matrix $\Gamma_{r, \mu_{z, n}, q}(z)$, where 
$\mu_{z, n}=n^{-1} \sum_{i=0}^{n-1} \delta_{f^{i}(z)}$ and $z \in M$. The proof of (2.3) is completed by proving the existence of a set $B$ of full $\mu$-measure such that for $z \in B$ and $x \in \operatorname{spt}(\mu), S_{r, \mu_{z, n}}(x)$ is defined for large $n$, and $\lim _{n \rightarrow \infty} S_{r, \mu_{z, n}}(x)=S_{r, \mu}(x)$ holds. In this case we have that $\lim _{n \rightarrow \infty} \Gamma_{r, \mu_{z, n}, q}(z)=\Gamma_{r, \mu, q}(z)$ for $q \in \operatorname{IN}$ and $z \in B \cap\left(\bigcap_{k=0}^{\infty} f^{-k}(\operatorname{spt}(\mu))\right)$ and by the continuity of the eigenvalues of a matrix with respect to its entries we get

$$
\lim _{n \rightarrow \infty} \alpha_{r, \mu_{z, n}, j}^{(q)}(z)=\alpha_{r, \mu, j}^{(q)}(z) \text { for } q \in \mathrm{IN}, j=1, \ldots, s
$$

which together with (2.5) gives (2.3).

Now we prove that $\mu$-a.e. $z$, and for $x \in \operatorname{spt}(\mu), S_{r, \mu_{z, n}}(x)$ is defined for large $n$, and $\lim _{n \rightarrow \infty} S_{r, \mu_{z, n}}(x)=S_{r, \mu}(x)$ holds. For the proof of existence and uniqueness of $S_{r, \mu_{z, n}}(x)$ we have to check that $\mu_{z, n} \in P(B(x, r))$ and $|f|_{p} \in L^{p}\left(\mu_{z, n} \mid B(x, r)\right)$ holds for large $n$ (see Lemma 1). Since $\operatorname{dim} \mu>s-1$ we have that $\mu \in P(B(x, r))$ for any $x \in \operatorname{spt}(\mu)$, and compactness of $\operatorname{spt}(\mu)$ implies $|f|_{p} \in L^{p}(\mu)$. Thus, it suffices to show that $\left\{\mu_{z, n}\right\}$ converges weakly to $\mu$ for $\mu$-a.e $z \in M$. When $f$ is a continuous dynamics, this fact is well known. It is not difficult to see that this is also true for any Borel measurable $\mu$-preserving dynamics: observing that the set of balls centered at points with rational coordinates and with rational radii are a countable basis for the usual topology of $\operatorname{IR}^{s}$, the weak convergence of $\left\{\mu_{z, n}\right\}$ to $\mu$ for $\mu$-a.e $z \in M$ follows from Theorem 2.2 in [4]. Let $B_{1}$ denote the set of full $\mu$-measure where the weak convergence of $\left\{\mu_{z, n}\right\}$ to $\mu$ holds. We prove that there is a set $B \subset B_{1}$ of full $\mu$-measure such that for any $z \in B$ and any $x \in \operatorname{spt}(\mu)$, $\lim _{n \rightarrow \infty} S_{r, \mu_{z, n}}(x)=S_{r, \mu}(x)$ holds, showing that any subsequence of $\left\{S_{r, \mu_{z, n}}(x)\right\}$ contains a subsequence which converges to $S_{r, \mu}(x)$. In order to do this we first prove that $\left\{S_{r, \mu_{z, n}}(x)\right\}$ is a bounded sequence. Using Lemma $\mathbf{1}$ we get

$$
\left\|S_{r, \mu_{z, n}}(x)\right\|_{2} \leq \frac{2 \mathcal{A}_{r, x, \mu_{z, n}}(0)}{h_{r, x, \mu_{z, n}}\left(T_{r, x, \mu_{z, n}}\right)} .
$$

We get an upper bound for $\left\{\left\|S_{r, \mu_{z, n}}(x)\right\|_{2}\right\}$ proving that for any $z \in B$,

$$
\lim _{n \rightarrow \infty} h_{r, x, \mu_{z, n}}\left(T_{r, x, \mu_{z, n}}\right)=h_{r, x, \mu}\left(T_{r, x, \mu}\right) \text { and } \lim _{n \rightarrow \infty} \mathcal{A}_{r, x, \mu_{z, n}}\left(\alpha_{n}\right)=\mathcal{A}_{r, x, \mu}(\alpha)
$$

holds where $T_{r, x, \nu}, \nu \in\left\{\mu, \mu_{z, n}\right\}$ is the linear map in $\mathcal{S}$ where the minimum value of the functional $h_{r, x, \nu}$ is attained, and $\left\{\alpha_{n}\right\}$ is any sequence in $\mathcal{L}_{s}$ with $\lim _{n \rightarrow \infty} \alpha_{n}=$ $\alpha$. We also shall use the last fact to prove (2.4). 
Let $C$ be the set of functions given by

$$
C:=\left\{g: g(y)=\chi_{B(x, r)}\left(|f(y)-t-\alpha(y-x)|_{p}\right)^{p}, x \in M, t \in M, \alpha \in \mathrm{IR}^{s^{2}}\right\} .
$$

Let $M^{*}$ and $\mathcal{L}_{s}^{*}$ be countable and dense subsets respectively in $M$ and $\operatorname{IR}^{s^{2}}$ and let

$C^{*}:=\left\{g: g(y)=\chi_{B\left(x^{*}, r\right)}\left(\left|f(y)-t^{*}-\alpha^{*}\left(y-x^{*}\right)\right|_{p}\right)^{p}, x^{*} \in M^{*}, t^{*} \in M^{*}, \alpha \in \mathcal{L}_{s}^{*}\right\}$.

We prove that there is a set $B_{2} \subset B_{1}$ of full $\mu$-measure such that for any $\varepsilon>0$, $z \in B_{2}$ and $g \in C$ there are a $g^{*} \in C^{*}$ and an $n_{0} \in$ IN such that

$$
\left|\int g(y) d \mu(y)-\int g^{*}(y) d \mu(y)\right|<\varepsilon \text { and }\left|\int g(y) d \mu_{z, n}(y)-\int g^{*}(y) d \mu_{z, n}(y)\right|<\varepsilon
$$

hold for $n>n_{0}$. We give the more involved proof of the second inequality. The first one can be obtained in an analogous way.

Let $B_{2}:=B_{1} \cap\left(\cap_{i=0}^{\infty} f^{-i}(\operatorname{spt}(\mu))\right), g(y):=\chi_{B(x, r)}\left(|f(y)-t-\alpha(y-x)|_{p}\right)^{p} \in C$ and $h(y):=\chi_{B\left(x^{*}, r\right)}\left(|f(y)-t-\alpha(y-x)|_{p}\right)^{p}$ where $x^{*} \in M^{*}$ is taken close to $x$. Then for $z \in B_{2}$,

$$
\begin{gathered}
\left|\left[\int g(y) d \mu_{z, n}(y)\right]^{1 / p}-\left[\int h(y) d \mu_{z, n}(y)\right]^{1 / p}\right| \leq \\
{\left[\int_{B(x, r) \Delta B\left(x^{*}, r\right)}\left(|f(y)-t-\alpha(y-x)|_{p}\right)^{p} d \mu_{z, n}(y)\right]^{1 / p} \leq} \\
K\left(\mu_{z, n}\left(\left(B(x, r) \Delta B\left(x^{*}, r\right)\right)\right)^{1 / p}\right.
\end{gathered}
$$

where the symbol $\Delta$ denotes the symmetric difference of sets and $K$ is a constant, depending on $\|\alpha\|_{2}$ and $|t|_{2}$. The existence of $K$ derives from the boundness of $\operatorname{stp}(\mu)$ and from the fact that every $y$ in the orbit of $z$ belongs to $\operatorname{spt}(\mu)$. Let $\left\{x_{k}\right\}$ be a sequence in $M^{*}$ such that $\lim _{k \rightarrow \infty} x_{k}=x$. The last inequality together with the fact that $\lim _{k \rightarrow \infty} \lim _{n \rightarrow \infty} \mu_{z, n}\left(\left(B(x, r) \Delta B\left(x_{k}, r\right)\right)=\mu(\partial B(x, r))=0\right.$, allows us to choose $x^{*} \in M^{*}$ and $n_{0} \in \mathrm{IN}$ such that

$$
\left|\left[\int g(y) d \mu_{z, n}(y)\right]^{1 / p}-\left[\int h(y) d \mu_{z, n}(y)\right]^{1 / p}\right| \leq \varepsilon / 2, \text { for } n>n_{0} .
$$

Taking $g^{*}(y) \in C^{*}$ where $g^{*}(y):=\chi_{B\left(x^{*}, r\right)}\left(\left|f(y)-t^{*}-\alpha^{*}\left(y-x^{*}\right)\right|_{p}\right)^{p}$ satisfies 


$$
\left|\left[\int h(y) d \mu_{z, n}(y)\right]^{1 / p}-\left[\int g^{*}(y) d \mu_{z, n}(y)\right]^{1 / p}\right|<\varepsilon / 2
$$

we get

$$
\left|\left[\int g(y) d \mu_{z, n}(y)\right]^{1 / p}-\left[\int g^{*}(y) d \mu_{z, n}(y)\right]^{1 / p}\right| \leq \varepsilon \text { for } n>n_{0},
$$

and the continuity of $t(y)=y^{p}$ completes the proof.

Inequalities given in (2.9), together with the fact that $C^{*}$ is a countable set of functions and Birkhoff theorem gives the existence of a set $B \subset B_{2}$ of full $\mu$-measure such that for any $z \in B$ and $g \in C$,

$$
\lim _{n \rightarrow \infty} \int g(y) d \mu_{z, n}(y)=\int g(y) d \mu(y)
$$

holds. Then, for any $z \in B, x \in f^{-1}(\operatorname{spt}(\mu))$, and any sequence $\left\{\alpha_{n}\right\}$ on $\mathcal{L}_{s}$ such that $\lim _{n \rightarrow \infty} \alpha_{n}=\alpha$,

$$
\lim _{n \rightarrow \infty} \mathcal{A}_{r, x, \mu_{z, n}}\left(\alpha_{n}\right)=\mathcal{A}_{r, x, \mu}(\alpha)
$$

holds (notice that the weak convergence of $\left\{\mu_{z, n}\right\}$ to $\mu$ does not permit us to obtain (2.10) because $f$ is not assumed to be a continuous dynamics). Taking $z \in B$ and $\alpha_{n}=0$ for all $n$ in (2.10), we can get an upper bound for $\left\{\mathcal{A}_{r, x, \mu_{z, n}}(0)\right\}$ in (2.7). Since $\lim _{n \rightarrow \infty} h_{r, x, \mu_{z, n}}\left(T_{r, x, \mu_{z, n}}\right)=h_{r, x, \mu}\left(T_{r, x, \mu}\right)>0$ holds (see part (i) of Lemma $\mathbf{1 . 2}$ in [11]), we also get a lower bound for $\left\{h_{r, x, \mu_{z, n}}\left(T_{r, x, \mu_{z, n}}\right)\right\}$ in (2.7). Then there is an $n_{0}>0$ such that the sequence $\left\{S_{r, \mu_{z, n}}(x)\right\}_{n>n_{0}}$ is contained in a compact set. Thus, any subsequence of $\left\{S_{r, \mu_{z, n}}(x)\right\}_{n>n_{0}}$ contains a convergent subsequence, which we also denote by $\left\{S_{r, \mu_{z, n}}(x)\right\}$. If $\lim _{n \rightarrow \infty} S_{r, \mu_{z, n}}(x)=S^{*}$ with $S^{*} \neq S_{r, \mu}(x)$ then

$$
\lim _{n \rightarrow \infty} \mathcal{A}_{r, x, \mu_{z, n}}\left(S_{r, \mu_{z, n}}(x)\right)=\mathcal{A}_{r, x, \mu}\left(S^{*}\right)>\mathcal{A}_{r, x, \mu}\left(S_{r, \mu}(x)\right)
$$

which follows from (2.10) together with the uniqueness of the minimum of the functional $\mathcal{A}_{r, x, \mu}$. The above inequality contradicts

$$
\lim _{n \rightarrow \infty} \mathcal{A}_{r, x, \mu_{z, n}}\left(S_{r, \mu_{z, n}}(x)\right) \leq \lim _{n \rightarrow \infty} \mathcal{A}_{r, x, \mu_{z, n}}\left(S_{r, \mu}(x)\right)=\mathcal{A}_{r, x, \mu}\left(S_{r, \mu}(x)\right),
$$

so that

$$
\lim _{n \rightarrow \infty} S_{r, \mu_{z, n}}(x)=S_{r, \mu}(x)
$$

holds, which completes the proof of (2.3).

We now prove (2.4). Let $R_{r}^{(q)}\left(S_{r, \mu}\right)=\frac{1}{q} \sum_{i=0}^{q-1} \frac{1}{\left(\mu\left(B\left(f^{i}(z), r\right)\right)\right)^{1 / p}} \mathcal{A}_{r, f^{i}(z), \mu}\left(S_{r, \mu}\left(f^{i}(z)\right)\right)$. By Theorem 2.1, $R_{r, \mu}\left(S_{r, \mu}\right)=\lim _{q \rightarrow \infty} R_{r}^{(q)}\left(S_{r, \mu}\right)$. Then it is sufficient to prove 


$$
\begin{gathered}
\lim _{n \rightarrow \infty} \frac{1}{\left(\mu_{z, n}\left(B\left(f^{i}(z), r\right)\right)\right)^{1 / p}} \mathcal{A}_{r, f^{i}(z), \mu_{z, n}}\left(S_{r, \mu_{z, n}}\left(f^{i}(z)\right)\right)= \\
\frac{1}{\left(\mu\left(B\left(f^{i}(z), r\right)\right)\right)^{1 / p}} \mathcal{A}_{r, f^{i}(z), \mu}\left(S_{r, \mu}\left(f^{i}(z)\right)\right), i \in \mathrm{IN}
\end{gathered}
$$

holds $\mu$-a.e. $z$ which follows from (2.11) and (2.10) for $x=f^{i}(z)$ together with the weak convergence of $\left\{\mu_{z, n}\right\}$ to $\mu$ taking $z \in B \cap\left(\cap_{k=0}^{\infty} f^{-k}(\operatorname{spt}(\mu))\right)$.

Proposition 1. Let $M$ be a Borel subset of $\mathrm{IR}^{s}$, and let $f: M \rightarrow M$ be a Borel measurable dynamics. Let $\mu$ be an $f$-invariant measure with compact support and $\operatorname{dim} \mu>s-1$. Then, the parameter

$$
R:=\lim \inf _{r \rightarrow 0} \frac{\log R_{r, \mu}\left(S_{r, \mu}\right)}{\log r}
$$

is defined and

(i) If $f$ is Hölder continuous of exponent $\varepsilon \mu$-a.e. (that is $\mu$-a.e. $x \in M,|f(y)-f(x)|_{2} \leq$ $L\left(|y-x|_{2}\right)^{\varepsilon}$ holds for $\mu$-a.e. $y \in M$ where $0<\varepsilon<1$ and $L$ is a positive constant), then $R \geq \varepsilon$.

(ii) If $f$ is Lipstchitz $\mu$-a.e. (that is $\mu$-a.e. $x \in M,|f(y)-f(x)|_{2} \leq L|y-x|_{2}$ holds for $\mu$-a.e. $y \in M$ where $L$ is a positive constant), then $R \geq 1$.

(iii) If $M$ is open and $f \in C^{1+\varepsilon} \mu$-a.e (that is $f \in C^{1}$ and it has Hölder continuous derivatives of exponent $\varepsilon$ for $\mu$-a.e.), then $R \geq 1+\varepsilon$.

Proof. By Theorem 2.1. we know that $R_{r, \mu}\left(S_{r, \mu}\right)$ is defined. Let $G$ be a point to matrix mapping such that $\|G(x)\|_{2} \in L^{1}(\mu)$. Since $S_{r, \mu}(x)$ is the best linear estimate in $L^{p}(\mu \mid B(x, r))$-norm for $f$ at $x$,

$$
R_{r, \mu}\left(S_{r, \mu}\right) \leq \int_{M} \frac{1}{(\mu(B(x, r)))^{1 / p}} \mathcal{A}_{r, x, \mu}(G(x)) d \mu(x)
$$

holds. Taking $G(x)=0$ in the last inequality we obtain (i) and (ii), and taking $G(x)=D f(x)$ we obtain (iii).

The next theorem shows how E.R.A. can be used to compute the Liapunov exponents of the tangent map when the dynamics is defined in a $d$-dimensional smooth submanifold $M$ of $\operatorname{IR}^{s}$ with $d \leq s$. Let $z \in \operatorname{spt}(\mu), i \in \operatorname{IN}, x_{i}=f^{i}(z)$ and $g_{i}=\Phi_{i+1} \circ f \circ \Phi_{i}^{-1}$ where $\left(U_{i}, \Phi_{i}\right)$ is the chart at $x_{i}$ defined in section 1.6. The algorithm acts by fitting the matrix $G_{r, n, i}(0)$ of the best linear estimate in 
$L^{p}\left(\nu_{n, i} \mid B(0, r)\right)$-norm of $g_{i}$ at the origin, where $\nu_{n, i}=\Phi_{i} \#\left(\mu_{z, n} \mid U_{i}\right)$. The exponents $\alpha_{r, n, j}^{(q)}(z), j=1, \ldots, d$ that E.R.A. gives, are the logarithms of the eigenvalues of the matrix $\Gamma_{r, n, q}(z):=\left(G_{r, n}^{(q) *} G_{r, n}^{(q)}\right)^{(2 q)^{-1}}$ where $G_{r, n}^{(q)}=G_{r, n, q-1}(0) G_{r, n, q-2}(0) \cdots$ $G_{r, n, 0}(0)$.

We say that $\mu$ is exact dimensional if

$$
\liminf r_{r \rightarrow 0} \frac{\log (\mu(B(x, r)))}{\log r}=\lim \sup _{r \rightarrow 0} \frac{\log (\mu(B(x, r)))}{\log r}=\alpha \mu \text {-a.e. }
$$

Theorem 2.3. Let $M$ be a d-dimensional submanifold of $\operatorname{IR}^{s}, f: M \rightarrow M$ and let $\mu$ be an $f$-invariant, ergodic and exact dimensional Borel probability measure with $\operatorname{dim} \mu>d-1$. Assume that there is an $\varepsilon>0$ such that $M$ is $C^{1+\varepsilon}, f \in C^{1+\varepsilon}(M)$ and $\int_{M} \log ^{+}(\|D f\|) d \mu<\infty$. For $p \in(1, \infty)$ and $r>0$, let $\alpha_{r, n, j}^{(q)}(z), j=1, \ldots, d$ be the estimates of the Liapunov exponents provided by the Eckmann and Ruelle algorithm from the orbit $O_{n}(z)$ and let $\lambda_{j}, j=1, \ldots, d$ be the $\mu$-a.e. constant Liapunov exponents for the tangent map $D f$. Then,

$$
\lim _{q \rightarrow \infty} \lim _{r \rightarrow 0} \lim _{n \rightarrow \infty} \alpha_{r, n, j}^{(q)}(z)=\lambda_{j}, j=1, \ldots, d
$$

for $\mu$-a.e. $z \in M$.

Proof. It is not difficult to prove that for each point $x \in M$ there exist a neighborhood $V_{x}$ in $\mathrm{IR}^{s}$ and a diffeomorphism $\Psi_{x}$ defined in $V_{x}$ such that the restriction of $\Psi_{x}$ to $V_{x} \cap M$ is given by $\Psi_{x}(y)=\Pi_{T_{x}(M)}(y-x)$ where $\Pi_{T_{x}(M)}$ denotes the orthogonal projection of $\operatorname{IR}^{s}$ into the tangent space $T_{x}(M)$ of $M$ at $x$. Thus, $\left(V_{x}, \Psi_{x}\right)$ provides a chart at $x$. This result is also true if we consider the orthogonal projection on any linear $d$-dimensional subspace $T$ such that $T^{\perp} \cap T_{x}(M)=\{0\}$, where $T^{\perp}$ denotes the orthogonal complement of $T$. Since $\operatorname{dim} \mu>d-1$, and $\left\{\mu_{z, n}\right\}$ converges weakly to $\mu$ for $\mu$-a.e. $z \in M$, we have that for $x_{i} \in \operatorname{spt}(\mu)$ and $r>0$, there is an $n_{0}>0$ such that for $n>n_{0}$ we can find $d$ vectors $v_{j}:=x_{i_{j}}-x_{i}$ with $x_{i_{j}} \in B\left(x_{i}, r\right) \cap O_{n}(z)$ spanning a $d$-dimensional linear subspace $T_{r, n, i}$. Moreover, since $x_{i_{j}} \in B\left(x_{i}, r\right) \cap M, j=1, \ldots, d$, for a sufficiently small $r$ and large $n, T_{r, n, i}^{\perp} \cap T_{x_{i}}(M)=\{0\}$ holds. Thus, we can choose the chart $\left(U_{i}, \Phi_{i}\right)$ at $x_{i} \in O_{n}(z)$ as described in section 1.6.

Since the hypotheses of Oseledec theorem hold, the Liapunov exponents of $D f$, which we have denoted by $\lambda_{j}, j=1, \ldots, d$, are defined and they are the logarithms of the eigenvalues of the $\mu$-a.e. $z$ well defined matrix $\Lambda_{z}:=\lim _{q \rightarrow \infty} \Lambda_{q}(z)$ where $\Lambda_{q}(z):=\left(D g^{(q) *} D g^{(q)}\right)^{(2 q)^{-1}}$ and $D g^{(q)}:=D g_{q-1}(0) D g_{q-2}(0) \cdots D g_{0}(0)$. 
By the continuity of the eigenvalues with respect to the entries of the matrix we have

$$
\lim _{q \rightarrow \infty} \lambda_{j}^{(q)}(z)=\lambda_{j}, j=1, \ldots, d
$$

$\mu$-a.e. where $\lambda_{j}^{(q)}(z), j=1, \ldots, d$ are the logarithms of the eigenvalues of $\Lambda_{q}(z)$.

Let $x_{i} \in O_{n}(z) \cap \operatorname{spt}(\mu)$. Since $\operatorname{dim} \mu>d-1$ and $\Phi_{i}$ is a diffeomorphism, we get that $\operatorname{dim} \nu_{i}>d-1$ where $\nu_{i}=\Phi_{i} \#\left(\mu \mid U_{i}\right)$. Then, $\nu_{i} \in P(B(0, r))$ holds. Since $g_{i}$ is a differentiable function we also get that $\left|g_{i}\right|_{p} \in L^{p}\left(\nu_{i} \mid B(0, r)\right)$ for small $r$, and by Lemma 1 the existence of the best linear estimate in $L^{p}\left(\nu_{i} \mid B(0, r)\right)$-norm for $g_{i}$ at the origin is guaranteed. We denote it by $G_{r, i}(0)$.

We now see that there is a set $A$ with $\mu(A)=1$ such that for all $z \in A$, and $i \in \mathrm{IN}, \lim _{r \rightarrow 0} G_{r, i}(0)=D g_{i}(0)$ holds. Results given in [11] ensure the existence of a set $Z_{i} \subset \Phi_{i}\left(U_{i}\right)$ of full $\nu_{i}$-measure such that $\lim _{r \rightarrow 0} G_{r, i}(a)=D g_{i}(a)$ holds for $a \in Z_{i}$. We need that for $z \in A, 0=\Phi_{i}\left(f^{i}(z)\right) \in Z_{i}$ holds for any $i \in$ IN. Notice that the charts $\Phi_{i}$ are depending on $z$. This is the reason for which we introduce a non depending on $z$ countable atlas $\left\{\left(V_{j}, \Psi_{j}\right)\right\}_{j \in \text { IN }}$ of $M$ which together with a change of charts shall allow us to get the result.

Let $\tau_{j}=\Psi_{j} \#\left(\mu \mid V_{j}\right), j \in \mathrm{IN}$. Since $\operatorname{dim} \tau_{j}>d-1$ we can apply Lemmas 3.1 and 3.2 in [11], so that for any $\sigma>0$ there exists a set $W_{j} \subset \Psi_{j}\left(V_{j}\right)$ of full $\tau_{j}$-measure such that for any $a \in W_{j}$ there are positive constants $K$ and $r_{0}$, depending on $a$, such that for any $\beta \in \mathcal{L}_{d}$ we get

$$
\|\beta\|_{2} \leq \frac{K}{r^{1+\sigma}\left(\tau_{j}(B(a, r))\right)^{1 / p}}\left[\int_{B(a, r)}\left(|\beta(y-a)|_{p}\right)^{p} d \tau_{j}(y)\right]^{1 / p}
$$

for $r<r_{0}$. Consider the set of full $\mu$-measure given by

$$
A:=\cap_{k=0}^{\infty} f^{-k}\left(\operatorname{spt}(\mu) \cap\left(\cup_{j=1}^{\infty} \Psi_{j}^{-1}\left(W_{j}\right)\right)\right) .
$$

Let $z \in A, i \in \mathrm{IN}$ and $x_{i}=f^{i}(z)$. Since $x_{i} \in A$, there is a $j \in \mathrm{IN}$ such that $x_{i} \in \Psi_{j}^{-1}\left(W_{j}\right) \cap \operatorname{spt}(\mu)$. Let $\left(U_{i}, \Phi_{i}\right)$ be the chart that the algorithm takes at $x_{i}$ and let $h_{i}: \Phi_{i}\left(U_{i} \cap V_{j}\right) \rightarrow \Psi_{j}\left(U_{i} \cap V_{j}\right)$ be the $C^{1+\varepsilon}$ map of change of charts given by $h_{i}:=\Psi_{j} \circ \Phi_{i}^{-1}$. Then, there is a constant $L$ such that

$$
\begin{gathered}
\left|\beta\left(h_{i}(y)-h_{i}(0)\right)\right|_{p} \leq\left|\beta\left(h_{i}(y)-h_{i}(0)-D h_{i}(0) y\right)\right|_{p}+\left|\beta D h_{i}(0) y\right|_{p} \leq \\
L\|\beta\|_{2}\left(|y|_{p}\right)^{1+\varepsilon}+\left|\beta D h_{i}(0) y\right|_{p} \text { for any } y \in \Phi_{i}\left(U_{i} \cap V_{j}\right)
\end{gathered}
$$


Using that for small $r, \tau_{j}\left|B\left(\Psi_{j}\left(x_{i}\right), r\right)=\left(h_{i} \# \nu_{i}\right)\right| B\left(\Psi_{j}\left(x_{i}\right), r\right)$ holds, a change of variable in the right hand of (2.14) taking $a=\Psi_{j}\left(x_{i}\right)$ and $\sigma<\varepsilon$, and (2.15) we get

$$
\begin{gathered}
\|\beta\|_{2} \leq \frac{K r^{-1-\sigma}}{\left(\tau_{j}\left(B\left(\Psi_{j}\left(x_{i}\right), r\right)\right)\right)^{1 / p}}\left[\int_{h_{i}^{-1}\left(B\left(h_{i}(0), r\right)\right)}\left(\left|\beta\left(h_{i}(y)-h_{i}(0)\right)\right|_{p}\right)^{p} d \nu_{i}(y)\right]^{1 / p} \leq \\
\left.\frac{K_{1} r^{-1-\sigma}}{\left(\tau_{j}\left(B\left(\Psi_{j}\left(x_{i}\right), r\right)\right)\right)^{1 / p}}\left[\left.\int_{h_{i}^{-1}\left(B\left(h_{i}(0), r\right)\right)}\left(\mid \beta D h_{i}(0) y\right)\right|_{p}\right)^{p} d \nu_{i}(y)\right]^{1 / p} \leq \\
\left.\frac{K_{1} r^{-1-\sigma}}{\left(\tau_{j}\left(B\left(\Psi_{j}\left(x_{i}\right), r\right)\right)\right)^{1 / p}}\left[\left.\int_{B(0, M r)}\left(\mid \beta D h_{i}(0) y\right)\right|_{p}\right)^{p} d \nu_{i}(y)\right]^{1 / p}
\end{gathered}
$$

for small $r$, where $K_{1}$ and $M$ are constants with $M>\left\|D h_{i}^{-1}\left(h_{i}(0)\right)\right\|_{2}$. Replacing $r$ with $r / M$ and taking $\beta=\left(G_{r, i}(0)-D g_{i}(0)\right) D h_{i}^{-1}\left(h_{i}(0)\right)$ in $(2.16)$, and using that $G_{r, i}(0)$ satisfies

$$
\left[\int_{B(0, r)}\left(\left|\left(G_{r, i}(0)-D g_{i}(0)\right) y\right|_{p}\right)^{p} d \nu_{i}(y)\right]^{1 / p} \leq 2\left[\int_{B(0, r)}\left(\left|g_{i}(y)-D g_{i}(0) y\right|_{p}\right)^{p} d \nu_{i}(y)\right]^{1 / p}
$$

and that $g_{i} \in C^{1+\varepsilon}$, we get

$$
\begin{aligned}
& \left\|G_{r, i}(0)-D g_{i}(0)\right\|_{2} \leq\left\|\left(G_{r, i}(0)-D g_{i}(0)\right) D h_{i}^{-1}\left(h_{i}(0)\right)\right\|_{2}\left\|D h_{i}(0)\right\|_{2} \leq \\
& \frac{K_{2} r^{-1-\sigma}}{\left(\tau_{j}\left(B\left(\Psi_{j}\left(x_{i}\right), \frac{r}{M}\right)\right)\right)^{1 / p}}\left[\int_{B(0, r)}\left(\left|\left(G_{r, i}(0)-D g_{i}(0)\right) y\right|_{p}\right)^{p} d \nu_{i}(y)\right]^{1 / p} \leq \\
& \frac{K_{3} r^{\varepsilon-\sigma}\left(\nu_{i}(B(0, r))\right)^{1 / p}}{\left(\tau_{j}\left(B\left(\Psi_{j}\left(x_{i}\right), \frac{r}{M}\right)\right)\right)^{1 / p}}=K_{3} r^{\varepsilon-\sigma}\left(\frac{\mu\left(\Phi_{i}^{-1}(B(0, r))\right)}{\mu\left(\Psi_{j}^{-1}\left(B\left(\Psi_{j}\left(x_{i}\right), \frac{r}{M}\right)\right)\right)}\right)^{1 / p},
\end{aligned}
$$

where $K_{i}, i=1,2,3$ are constants. Using that for any diffeomorhism $\phi, B\left(x, \frac{r}{l_{1}}\right) \subset$ $\phi^{-1}(B(\phi(x), r)) \subset B\left(x, l_{2} r\right)$ holds, where $l_{1}$ and $l_{2}$ are constants with $l_{1}>\|D \phi(x)\|_{2}$ and $l_{2}>\left\|D \phi^{-1}(\phi(x))\right\|_{2}$ together with the fact that $\mu$ is exact dimensional, $\sigma<\varepsilon$, and (2.17) we get that $\left\|G_{r, i}(0)-D g_{i}(0)\right\|_{2}=o\left(r^{\varepsilon-\alpha}\right)$ where $\alpha$ is any constant with $0<\alpha<\varepsilon$. 
We now show that $\mu$-a.e. $z, G_{r, n, i}(0)$ is defined and $\lim _{n \rightarrow \infty} G_{r, n, i}(0)=G_{r, i}(0)$. Let $B$ be the set of full measure where the weak convergence of $\left\{\mu_{z, n}\right\}$ to $\mu$ holds. Then for any $z \in B$ and $i \in \mathrm{IN}$, we also have the weak convergence of $\left\{\nu_{n, i}\right\}$ to $\nu_{i}$. Using that $\nu_{i} \in P(B(0, r))$ and $\left|g_{i}\right|_{p} \in L^{p}\left(\nu_{i} \mid B(0, r)\right)$ for small $r$, we get that $\nu_{n, i} \in P(B(0, r))$ and $\left|g_{i}\right|_{p} \in L^{p}\left(\nu_{n, i} \mid B(0, r)\right)$ for small $r$ and large $n$, and again by Lemma $1 G_{r, n, i}(0)$ is defined for small $r$ and large $n$. Using the argument given in Theorem 2.2 when proving $\lim _{n \rightarrow \infty} S_{r, \mu_{z, n}}(x)=S_{r, \mu}(x)$, we get $\lim _{n \rightarrow \infty} G_{r, n, i}(0)=G_{r, i}(0)$ for any $z \in B$ (notice that in this case the continuity of $g_{i}$ simplifies the proof). Thus, we have obtained $\lim _{r \rightarrow 0} \lim _{n \rightarrow \infty} G_{r, n, i}(0)=$ $\lim _{r \rightarrow 0} G_{r, i}(0)=D g_{i}(0)$ for $i \in \mathrm{IN}$ and $z \in A \cap B$. Hence,

$$
\lim _{r \rightarrow 0} \lim _{n \rightarrow \infty} G_{r, n}^{(q)}(0)=D g^{(q)} \text { for } q \in \mathrm{IN}
$$

$\mu$-a.e.z, and by the continuity of the eigenvalues of the matrix with respect to its entries

$$
\lim _{r \rightarrow 0} \lim _{n \rightarrow \infty} \alpha_{r, n, j}^{(q)}(z)=\lambda_{j}^{(q)}(z), j=1, \ldots, d, q \in \mathrm{IN}
$$

which together with (2.13) gives (2.12).

\section{Concluding remarks.}

Remark 1. Eckmann and Ruelle conjectured that an ergodic measure invariant under a $C^{2}$-diffeomorphism with non zero Liapunov exponent is regular and exact dimensional (see [6]). This conjecture has been proved in ([3]) for a compactly supported Borel probability measure, with non-zero Liapunov exponents and invariant under a $C^{1+\varepsilon}$ diffeomorphism of a smooth Riemannian manifold.

Remark 2. The result of Theorem 2.3 can be obtained for a non exact dimensional measure if $f \in C^{1+\varepsilon}$ with $\varepsilon>\frac{\operatorname{Dim} \mu-\operatorname{dim} \mu}{\operatorname{dim} \mu-d+1}+\frac{\operatorname{Dim} \mu-\operatorname{dim} \mu}{p}$ where Dim $\mu$ denotes the packing dimension of $\mu$ (see [19]). The proof is like in Theorem $\mathbf{2 . 3}$ considering $\sigma>(\operatorname{Dim} \mu-\operatorname{dim} \mu) /(\operatorname{dim} \mu-d+1)$ in (2.14) and taking into account in (2.17) that $\frac{\mu\left(B\left(x_{i}, r\right)\right)}{\mu\left(B\left(x_{i}, r / k\right)\right)}<k^{\alpha_{2}} r^{\alpha_{1}-\alpha_{2}}$ holds for $r$ small and any $\alpha_{1}$ and $\alpha_{2}$ with $\alpha_{1}<\operatorname{dim} \mu$ and $\alpha_{2}>\operatorname{Dim} \mu$.

In this theorem we can also assume that $M$ is an open subset of $\operatorname{IR}^{s}$ and $\operatorname{dim} \mu>$ $s-1$. Then the result is obtained if $f$ is locally $C^{1+\varepsilon} \mu$-a.e. where $\varepsilon>\frac{\operatorname{Dim} \mu-\operatorname{dim} \mu}{\operatorname{dim} \mu-d+1}$.

Remark 3. The assumption $\operatorname{dim} \mu>k-1$ made in the previous theorems, for $k=s$ in Theorems $\mathbf{2 . 1}$ and $\mathbf{2 . 2}$, and $k=d$ in Theorem 2.3, seems to be a natural 
condition which ensures that the dynamics does not take place in a submanifold of dimension smaller than $k$, in which case the existence of the $k$ Liapunov exponents computed from the best linear estimates in $\mathcal{L}_{k}$ is not guaranteed.

Remark 4. We do not know if, under the hypotheses of Theorem $\mathbf{2 . 3}$ when $d=s$, the $L_{r}^{p}$-Liapunov exponents converge to the Liapunov exponents of $D f$ when $r$ tends to zero. Although $\lim _{r \rightarrow 0} S_{r, \mu}(x)=D f(x) \mu$-a.e, the rate of convergence required by the available results on perturbation of infinite products of matrices (see [15]), is not guaranteed.

\section{References}

[1] Abarbanel H., R. Brown \& M.B. Kennel. Liapunov Exponents in Chaotic Systems: their Importance and their Evaluation using Observed Data. Int. J. of Mod. Physics B, 5, 9, (1991), 1347-75.

[2] Brown R., Bryant P. \& Abarbanel H. Computing the Lyapunov spectrum of a dynamical system from an observed time series. Physical Review A, 43, 6, (1991) 2787-2806.

[3] L. Barreira, Y. Pesin \& J. Schmeling. On the Pointwise Dimension of Hyperbolic Measures: A Proof of the Eckmann-Ruelle Conjecture. Electronic Research Announcements of the American Mathematical Society, 2, 1, (1996), 69-72.

[4] P. Billingsley. Convergence of Probability Measures, John Wiley, 1968.

[5] J.P.R. Christensen. Topology and Borel Structure, NorthHolland/American Elsevier,1974.

[6] J.P. Eckmann \& D. Ruelle. Ergodic Theory of Chaos and Strange Attractors, Rev. Mod. Phys., 57, (1985), 617-656.

[7] J.P. Eckmann, S.O. Kamphorst, D. Ruelle \& S. Ciliberto. Liapunov Exponents from Time Series, Physical Review A, 34, 6, (1986), 4971-4979.

[8] P. Grassberger. An Optimized Box-Assisted Algorithm for Fractal Dimensions, Phys. Lett. A, 148, (1990), 63-68. 
[9] P. Henrici. Applied and Computational Complex Analysis, Vol 1, Wiley, New York, 1974.

[10] P. Mattila. Geometry of Sets and Measures in Euclidean Spaces. Fractals and Rectifiability, Cambridge University Press, 1995.

[11] M.E. Mera \& M. Morán. $L^{p}(\mu)$-Estimation of Tangent Maps (Preprint).

[12] M.E. Mera \& M. Morán. Estimation of Liapunov exponents from time series (manuscript in preparation).

[13] V.I. Oseledec. A Multiplicative Ergodic Theorem. Liapunov Characteristic Exponents, Trans. Moscow Math. Soc., 19, (1968), 179-231.

[14] R.T. Rockafellar. Convex Analysis. Princeton University Press, (1972)

[15] D. Ruelle. Ergodic Theory of Differentiable Dynamical Systems, Publ. Math. IHES, 50, (1979), 275-307.

[16] M. Sano \& Y. Sawada. Measurement of the Liapunov Spectrum from a Chaotic Time Series. Phys. Rev. Lett. 55, 10, (1985), 1082-85.

[17] S. Sato, M. Sano \& Y. Sawada. Practical Methods of Measuring the Generalized Dimension and the Largest Liapunov Exponent in High Dimensional Chaotic Systems, Prog. Theor. Phys., 77, (1987), 1.

[18] F. Takens. Detecting Strange Attractors in Turbulence, Dynamical Systems and Turbulence. Lectures Notes in Mathematics, 898, 396, (1981).

[19] C. Tricot. Two definitions of Fractional Dimension, Math. Proc. Camb. Phil. Soc., 91, (1982), 57-74.

[20] P. Walters. An Introduction to Ergodic Theory. Springer Verlag, 1982.

[21] A. Wolf, J.B. Swift, H.L. Swinney \& A. Vastano. Determining Liapunov Exponents from Time Series, Physica D, 16, (1984), 285-317. 